\title{
Predictive Torque Control With Fixed Switching Frequency for Induction Motor Drives
}

\author{
Jose A. Riveros \\ Facultad de Ingeniería \\ Universidad de Talca \\ Curicó, Chile \\ jriveros@utalca.cl
}

\author{
Marco Rivera \\ Facultad de Ingeniería \\ Universidad de Talca \\ Curicó, Chile \\ marcoriv@utalca.cl
}

\author{
Consuelo Rodríguez \\ Facultad de Ingeniería \\ Universidad de Talca \\ Curicó, Chile \\ crodriguez12@alumnos.utalca.cl
}

\author{
Michael Galea \\ PEMC Research Group \\ The University of Nottingham \\ Ningbo, China \\ Michael.Galea@nottingham.edu.cn
}

\author{
Giampaolo Buticchi \\ PEMC Research Group \\ The University of Nottingham \\ Ningbo, China \\ Giampaolo.Buticchi@nottingham.edu.cn
}

\author{
Patrick Wheeler \\ PEMC Research Group \\ The University of Nottingham \\ Nottingham, UK, and Ningbo, China \\ Pat.Wheeler@nottingham.ac.uk
}

\begin{abstract}
Typical predictive torque control is one of the most considered high-performance methods for variable-speed applications. This scheme is characterised by a flexible architecture that provides a fast torque/flux response, while its operation reports variable switching frequency as one of the main issues. This work presents a redesigned technique to overcome the described drawback. The strategy applies two active voltage space vectors with an appropriate sequence at every sampling period to produce a constant number of commutations. The dwell times are computed with the conventional cost function and a novel optimisation process to obtain the proper switching combination. Simulation tests at transient- and steady-state demonstrate the quick dynamic response of this proposal, whereas a performance comparison respect to the conventional implementation illustrates the achieved enhancements and mitigation trade-off.

Index Terms-Predictive control, torque control, variable speed drives.
\end{abstract}

\section{INTRODUCTION}

Direct torque/flux control strategies have been proposed for electric motor drive applications where a quick dynamic response in a wide speed range is required [1,2]. Electric traction/propulsion, more-electric aircraft actuators are some examples of the niche of uses of this scheme. The architecture of this proposal is commonly composed of external controllers, which generate the electromagnetic torque and stator flux references [2]. Then, the target of the technique is to track these command values injecting currents below the rated limit and with the minimal distortion possible [3].

One of the most promoted methods with the aforementioned design is predictive torque control (PTC) [4]. This strategy was introduced as an alternative to the widely studied direct torque control (DTC) technique. DTC is a simple and robust controller for the speed regulation based on two hysteresis comparators, the stator flux position and a lookup table for the selection of the adequate voltage vector [5]. The architecture of DTC is inflexible to cover additional criteria in the control law [6]. On the other hand, PTC has been proposed in applications where a higher number of degrees of freedom are regulated [7]. Additionally, more accurate models can be easily implemented with PTC, attaining better performance than the DTC algorithm in terms of torque/flux ripple [6]. However, a higher computational burden is required by PTC.

The classic PTC scheme for induction motor drives is composed of a predictive torque/flux model, a cost function and an optimisation stage [8]. The first one is used to predict the behaviour of the regulated variables. The control law is defined in the cost function with weighted errors of torque and flux respect to their reference values [9]. This last is usually optimised by evaluating a finite set of switching states of the voltage source inverter (VSI) and selecting the alternative that minimises the cost function. The implementation described is easy to understand but reported operation with variable switching frequency [4]. This feature is undesired in power electronics applications due to the complex and expensive power filter required for the mitigation [10].

The power spectrum drawback of predictive control strategies was already analysed in the literature [11]. The stationary reference frame is organised in sectors compounded of two active vectors. The proper application of these lasts with the zero voltage vectors produces a symmetrical switching pattern with a fixed number of commutations according to the space vector modulation (SVM) theory [12]. Then, the simpler solution is to include a modulator in the predictive control architecture to attain fixed switching frequency [13]. Nevertheless, the advantages of predictive control methods are sacrificed to attain the desired feature with higher complexity.

An alternative predictive control scheme for current regulation with fixed switching frequency was reported in [14]. A cost function for the evaluation of the sectors was proposed with the current tracking errors and the dwell times of their respective vectors. The optimum cost function of each sector can be calculated by solving a minimisation problem with the voltage vectors and the time restriction. Finally, the pair of 
vectors with their respective dwell times of the sector that achieve the global minimum is selected and applied during the next sampling period. In this manner, the predictive scheme is used to determine the sector, switching states and times.

A PTC strategy with fixed switching frequency for induction motor drives is introduced in this work. The scheme is derived following the same principles employed in [14], but considering the electromechanical system and the torque/flux control. Thus, a cost function for the evaluation of the sectors incorporating the torque and flux deviations of two voltage vectors with their respective dwell times influences is developed for the optimisation process. Next, optimal solutions of the sectors are determined offline and employed in a global minimisation procedure to obtain the optimum combination of voltage vectors along with their application times during a sampling period. The described process also includes overcurrent protection. The traditional seven-segment switching pattern [12] is implemented to ensure a constant number of commutations, whereas the resulted switching and sampling frequencies are the same. The achieved controller provides a quick dynamic response with the desired power spectrum.

The paper is structured as follows. Section II overviews the induction motor drive model. Then, the new PTC strategy is described in Section III. Next, Section IV presents the simulation tests and comparison respect the classic PTC. Finally, the achievements are summarised in the last section.

\section{Model of the Electromechanical System}

The AC drive considered in this work comprises an induction motor with star-connected stator windings and supplied by a two-level VSI, see Fig. 1. For the sake of simplifying the derivation of the control strategy, the system is analysed in the stationary reference frame $\alpha-\beta$. Thus, the stator voltage space vectors $\mathbf{v}_{\mathbf{s}}$ with invariant magnitude can be computed from the switching states $S_{n}(n=\{1,2,3\})$ and the dc-link voltage $V_{d c}$ as follows:

$$
\mathbf{v}_{\mathbf{s}}=v_{s \alpha}+\mathbf{j} \cdot v_{s \beta}=\frac{2}{3} V_{d c}\left[S_{1}+S_{2} \cdot \mathbf{a}+S_{3} \cdot \mathbf{a}^{2}\right]
$$

being $\mathbf{a}=e^{\mathbf{j} 2 \pi / 3}$ and $\mathbf{j}$ the imaginary unit. The switching state $S_{n}$ is 1 when the upper power switch of the leg $n$ is activated,

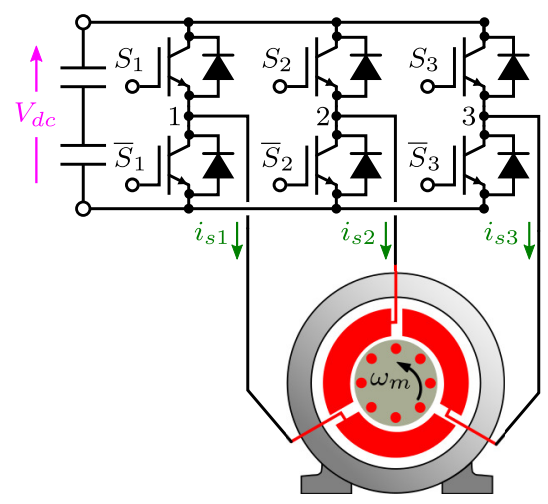

Fig. 1. Diagram of the induction motor drive

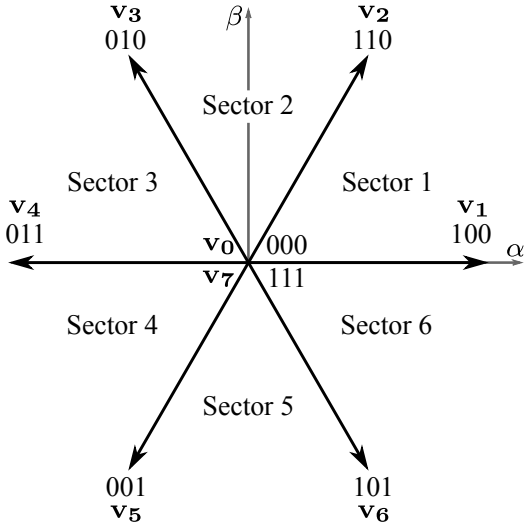

Fig. 2. Projections of the voltage space vectors in the $\alpha-\beta$ plane.

and 0 if the bottom one is turned on. The transformation (1) generates six active (magnitude $2 V_{d c} / 3$ ) and two zero voltage space vectors ([ $\left[\begin{array}{lll}0 & 0 & 0\end{array}\right]$ and $\left.\left[\begin{array}{lll}1 & 1 & 1\end{array}\right]\right)$, which are organised in six sectors, as indicates Fig. 2.

The state-space representation of the electromagnetic model of the induction machine in the stationary reference frame is described as follows [15]:

$$
\begin{aligned}
\frac{d \mathbf{x}}{d t} & =A \cdot \mathbf{x}+B \cdot \mathbf{u} \\
\mathbf{y} & =C \cdot \mathbf{x} \\
\mathbf{x} & =\left[\begin{array}{llll}
i_{s \alpha} & i_{s \beta} & \Psi_{r \alpha} & \Psi_{r \beta}
\end{array}\right]^{T} \\
\mathbf{u} & =\left[\begin{array}{llll}
v_{s \alpha} & v_{s \beta} & 0 & 0
\end{array}\right]^{T} \\
\mathbf{y} & =\left[\begin{array}{llll}
i_{s \alpha} & i_{s \beta} & \Psi_{s \alpha} & \Psi_{s \beta}
\end{array}\right]^{T}
\end{aligned}
$$

being $i$ and $\Psi$ the current and flux linkage, respectively, while the subscripts $s$ and $r$ designate the stator and rotor variables. The superscript $T$ denotes the transpose operator. The statespace vector $\mathbf{x}$ is composed of the stator current and the rotor flux in the $\alpha-\beta$ plane, while the output vector $\mathbf{y}$ is defined with the stator current and flux. The matrices employed in (2) are summarised in (3).

$$
\begin{aligned}
A & =\left[\begin{array}{cccc}
-\frac{1}{\tau_{\sigma}} & 0 & \frac{(1-\sigma)}{\sigma \cdot L_{m} \cdot \tau_{r}} & \frac{(1-\sigma)}{\sigma \cdot L_{m}} \omega_{r} \\
0 & -\frac{1}{\tau_{\sigma}} & -\frac{(1-\sigma)}{\sigma \cdot L_{m}} \omega_{r} & \frac{(1-\sigma)}{\sigma \cdot L_{m} \cdot \tau_{r}} \\
\frac{L_{m}}{\tau_{r}} & 0 & -\frac{1}{\tau_{r}} & -\omega_{r} \\
0 & \frac{L_{m}}{\tau_{r}} & \omega_{r} & -\frac{1}{\tau_{r}}
\end{array}\right] \\
B & =\left[\begin{array}{cccc}
-\frac{1}{\sigma \cdot L_{s}} & 0 & 0 & 0 \\
0 & -\frac{1}{\sigma \cdot L_{s}} & 0 & 0 \\
0 & 0 & 0 & 0 \\
0 & 0 & 0 & 0
\end{array}\right] \\
C & =\left[\begin{array}{cccc}
1 & 0 & 0 & 0 \\
0 & 1 & 0 & 0 \\
\sigma \cdot L_{s} & 0 & \kappa_{r} & 0 \\
0 & \sigma \cdot L_{s} & 0 & \kappa_{r}
\end{array}\right]
\end{aligned}
$$

The electromagnetic parameters of the induction machine used in this model are the magnetising inductance $\left(L_{m}\right)$, the stator and rotor inductances ( $L_{s}$ and $L_{r}$, respectively), the stator and rotor resistances ( $R_{s}$ and $R_{r}$, respectively). The mechanical 
and electrical speeds ( $\omega_{m}$ and $\omega_{r}$, respectively) verify the identity $\omega_{r}=p \cdot \omega_{m}$, being $p$ the pair of magnetic poles of the machine. The matrix components are defining with the following constants:

$$
\begin{aligned}
\sigma & =1-\frac{L_{m}^{2}}{L_{s} \cdot L_{r}} ; \kappa_{r}=L_{m} / L_{r} \\
\tau_{s} & =L_{s} / R_{s} ; \tau_{r}=L_{r} / R_{r} \\
\frac{1}{\tau_{\sigma}} & =\frac{1}{\sigma \cdot \tau_{s}}+\frac{(1-\sigma)}{\sigma \cdot \tau_{r}}
\end{aligned}
$$

The mechanical model of the system is ruled by the following differential equation and electromagnetic torque identity:

$$
\begin{aligned}
& \frac{d \omega_{m}}{d t}=\frac{1}{J_{m}}\left(T_{e}-T_{L}-B_{m} \cdot \omega_{m}\right) \\
& T_{e}=\frac{3}{2} p \cdot \Psi_{\mathbf{s}} \times \mathbf{i}_{\mathbf{s}}=\frac{3}{2} p \cdot\left(\Psi_{s \alpha} \cdot i_{s \beta}-\Psi_{s \beta} \cdot i_{s \alpha}\right)
\end{aligned}
$$

where the electromagnetic and load torques are labelled with $T_{e}$ and $T_{L}$, respectively. The inertia and friction coefficients are represented by $J_{m}$ and $B_{m}$, respectively.

The state-space representation of (2) should be discretised in order to be implemented in a digital controller and to derive the predictive model. The discrete-time model with a $T_{s}$ sampling period is the following $[7,15]$ :

$$
\begin{aligned}
\mathbf{x}(k+1) & =A_{d} \cdot \mathbf{x}(k)+B_{d} \cdot \mathbf{u}(k) \\
\mathbf{y}(k+1) & =C \cdot \mathbf{x}(k+1) \\
A_{d} & \approx I_{4}+A \cdot T_{s} \\
B_{d} & \approx B \cdot T_{s}
\end{aligned}
$$

being $I_{4}$ the $4 \times 4$ identity matrix. Notice that the discrete-time output vector $\mathbf{y}(k+1)$ is also computed with the continuoustime matrix $C$. The digital model (6) and the electromagnetic torque equation of (5) are employed by the PTC scheme in the predictive model.

\section{PTC WITH FIXED SWITCHING FREQUENCY}

The introduced PTC scheme uses the same principles established in the controller presented in [14]. However, a different power converter and a more complex system are considered in this work. The predictive model of the system is described in the previous section, whereas the cost function, switching strategy and optimisation process are detailed as follows.

\section{A. Cost Function}

The objective function $G$ used in this work for the evaluation of the voltage vector $\mathbf{v}_{\mathbf{m}}(m \in\{0,1, \ldots, 7\})$ is the traditional sum of the predicted torque and flux deviations respect to their references ( $T_{e}^{*}$ and $\Psi_{s}^{*}$, respectively) and normalised with the nominal values ( $T_{n}$ and $\Psi_{n}$, respectively)

\begin{tabular}{|c|c|c|c|c|c|c|c|}
\hline Segment & 1 & 2 & 3 & 4 & 5 & 6 & 7 \\
\hline Time & $\delta_{0} / 4$ & $\delta_{1} / 2$ & $\delta_{2} / 2$ & $\delta_{0} / 2$ & $\delta_{2} / 2$ & $\delta_{1} / 2$ & $\delta_{0} / 4$ \\
\hline Sequence & \multirow{7}{*}{$\mathrm{v}_{0}$} & $\mathbf{u}_{1}$ & $\mathbf{u}_{2}$ & \multirow{7}{*}{$v_{7}$} & $\mathbf{u}_{2}$ & $u_{1}$ & \multirow{7}{*}{$\mathrm{v}_{0}$} \\
\hline Sector 1 & & $\mathrm{v}_{1}$ & $\mathrm{v}_{2}$ & & $\mathbf{v}_{2}$ & $v_{1}$ & \\
\hline Sector 2 & & $v_{3}$ & $\mathbf{v}_{2}$ & & $\mathbf{v}_{2}$ & $v_{3}$ & \\
\hline Sector 3 & & $v_{3}$ & $v_{4}$ & & $\mathbf{v}_{4}$ & $v_{3}$ & \\
\hline Sector 4 & & $v_{5}$ & $\mathrm{v}_{4}$ & & $\mathrm{v}_{4}$ & $v_{5}$ & \\
\hline Sector 5 & & $v_{5}$ & $v_{6}$ & & $v_{6}$ & $\mathrm{v}_{5}$ & \\
\hline Sector 6 & & $\mathrm{v}_{1}$ & $v_{6}$ & & $v_{6}$ & $\mathrm{v}_{1}$ & \\
\hline
\end{tabular}
as indicated (7).

$$
G\left(\mathbf{v}_{\mathbf{m}}\right)=\frac{\left(T_{e}^{*}-T_{e}(k+1)\right)^{2}}{T_{n}^{2}}+\gamma \frac{\left(\Psi_{s}^{*}-\left|\Psi_{\mathbf{s}}\right|(k+1)\right)^{2}}{\Psi_{n}^{2}}
$$

being $T_{e}(k+1)$ and $\left|\boldsymbol{\Psi}_{\mathbf{s}}\right|(k+1)$ the predicted variables for the $(k+1)$ sampling period, whereas $\gamma$ is the weighting factor of the flux tracking error.
TABLE I

Seven-Segment Switching Strategy

\section{B. Switching Strategy}

The application of two active $\left(\mathbf{u}_{1}\right.$ and $\left.\mathbf{u}_{2}\right)$ and the zero $\left(\mathbf{v}_{\mathbf{0}}\right.$ and $\mathbf{v}_{\mathbf{7}}$ ) voltage vectors within a control period is required to achieve a fixed switching frequency. Hence, the problem can be analysed with the sectors of the $\alpha-\beta$ plane, see Fig. 2, following the SVM approach [12]. The normalised dwell periods ( $T_{s}$ is the base time) of $\mathbf{u}_{\mathbf{1}}$ and $\mathbf{u}_{2}$ are $\delta_{1}$ and $\delta_{2}$, respectively. The remaining time to complete the sampling period $\left(\delta_{0}=1-\delta_{1}-\delta_{2}\right)$ is equally distributed between the zero voltage vectors $\mathbf{v}_{\mathbf{0}}$ and $\mathbf{v}_{\mathbf{7}}$. The seven-segment pattern is attained with the sequence $\mathbf{v}_{0}-\mathbf{u}_{1}-\mathbf{u}_{\mathbf{2}}-\mathbf{v}_{\mathbf{7}}-\mathbf{u}_{\mathbf{2}}-\mathbf{u}_{\mathbf{1}}-\mathbf{v}_{\mathbf{0}}$. Table I summarises the vectors and dwell times during a sampling period for the six sectors.

\section{Optimisation}

The optimisation process is one of the main contributions of this work. Traditionally, this part is performed with an exhaustive survey of the 7 discrete switching states, and the one that minimises the cost function is applied during the next control period [8]. The proposed strategy should analyse the behaviour in the sectors with the combination of three voltage vectors [14]. Thus, the goal is to find the dwell times of the active vectors of Table I for each sector that provide the best reference tracking in this region and comply the time constraint. The following problem is proposed in the six sectors by using the cost function (7):

$$
\begin{aligned}
& F(\text { sector })=G_{1} \cdot \delta_{1}^{2}+G_{2} \cdot \delta_{2}^{2}+G_{0} \cdot \delta_{0}^{2} \\
& \delta_{1}+\delta_{2}+\delta_{0}=1 \\
& G_{m}=G\left(\mathbf{u}_{\mathbf{m}}\right) ; m=\{0,1,2\}
\end{aligned}
$$

The dwell times can be obtained offline with multivariable optimisation methods, to yield the next solution [14]:

$$
\begin{aligned}
& \frac{1}{\lambda}=\frac{1}{G_{1}}+\frac{1}{G_{2}}+\frac{1}{G_{0}} \\
& \delta_{1, \mathrm{opt}}=\lambda / G_{1} ; \delta_{2, \mathrm{opt}}=\lambda / G_{2} ; \delta_{0, \mathrm{opt}}=\lambda / G_{0} \\
& F_{\mathrm{opt}}=G_{1} \cdot \delta_{1, \mathrm{opt}}^{2}+G_{2} \cdot \delta_{2, \mathrm{opt}}^{2}+G_{0} \cdot \delta_{0, \mathrm{opt}}^{2}
\end{aligned}
$$

Finally, a global minimisation algorithm is proposed. This surveys the optimum solutions of the sectors and selects the minimum $F_{\text {opt }}$. Additionally, in this step the solutions computed with (9) that generate a predicted $\alpha-\beta$ stator current higher than the nominal $\left(I_{n}\right)$ are penalised (overcurrent 
protection [4]). The predicted magnitude of the stator current of the offline solution (9), $\left|\mathbf{i}_{\mathbf{s}}^{\mathbf{p}}\right|$, is calculated online with the predictive model by using the average voltage synthesised with the vectors $\mathbf{u}_{1}$ and $\mathbf{u}_{\mathbf{2}}$ of the considered sector and the dwell times $\delta_{1, \mathrm{opt}}$ and $\delta_{2, \mathrm{opt}}\left(\mathbf{u}_{\mathbf{1}} \cdot \delta_{1, \mathrm{opt}}+\mathbf{u}_{\mathbf{2}} \cdot \delta_{2, \mathrm{opt}}\right)$. Then, the last step evaluates the following function:

$$
G_{\text {global }}(\text { sector })=F_{\text {opt }}(\text { sector })+I_{\text {prot }} \cdot\left(\left|\mathbf{i}_{\mathbf{s}}^{\mathbf{p}}\right|(\text { sector })>I_{n}\right)
$$

being $I_{\text {prot }}$ the penalisation value, and it only affect the solutions of (9) that overpass the rated current by using the logic comparison operator indicated in (10).

\section{Summary of the Strategy}

A scheme of the proposed PTC technique is depicted in Fig. 3. A proportional-integral (PI) controller generates the electromagnetic torque reference $T_{e}^{*}$ with the speed error $\left(\omega_{m}^{*}-\omega_{m}\right.$, being $\omega_{m}^{*}$ the target value). The reference stator flux $\Psi_{s}^{*}$ is set to the nominal magnitude $\Psi_{n}$ for the operation in the rated speed region. The measurements (stator current, speed and dc-bus voltage) are used by the predictive model (6) to estimate the future response of the regulated variables. The last part supports the global optimisation stage; which surveys the six sectors evaluating the voltage vectors of this region (see $\mathbf{u}_{1}$ and $\mathbf{u}_{2}$ of Table I) with (7), computing the objective function $F_{\mathrm{opt}}$ with the offline solution of (9) and penalising the $\alpha-\beta$ stator currents with magnitudes higher than $I_{n}$ like in [4]. The optimum sector is selected in this manner, and the VSI is controlled with the symmetrical switching pattern strategy to achieve the operation with fixed switching frequency.

\section{RESUlTS}

The PTC shown in Fig. 3 and the electromechanical system are implemented in the Matlab/Simulink environment to conduct the validation tests. Transient- and steady-state responses, as well as a performance comparison with the classic PTC [8] are presented in this section. The parameters used in this process are summarised in Table II, including the proportional and integral constants of the PI controller $\left(K_{p}\right.$ and $K_{i}$, respectively). The flux weighting factor $\gamma$ was tuned with a trial-error procedure, and the constant $I_{\text {prot }}$ is set to 100 . Notice that the tuning of weighting factors is still a challenge like in the classical scheme.

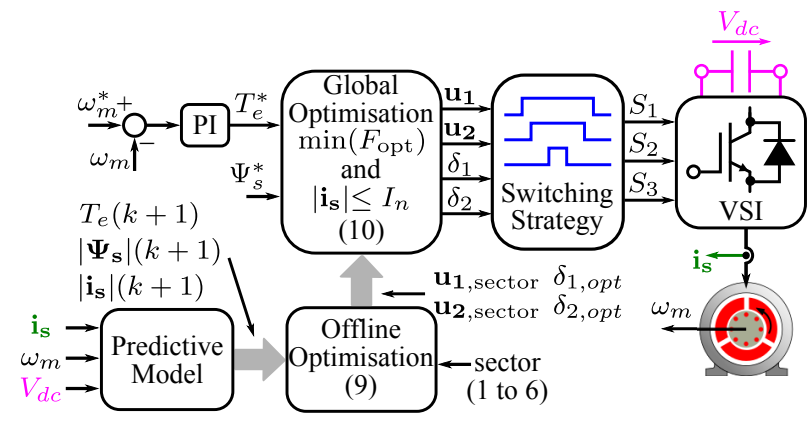

Fig. 3. Diagram of the proposed PTC scheme with fixed switching frequency operation.
TABLE II

SIMULATION PARAMETERS

\begin{tabular}{|l|c|c|}
\hline Parameter & Unit & Value \\
\hline$L_{m}, L_{s}, L_{r}$ & $(\mathrm{~m} \mathrm{H})$ & $282.0,285.9,285.9$ \\
\hline$R_{s}, R_{r}$ & $(\Omega)$ & $1.35,7.20$ \\
\hline$p$ & & 2.0 \\
\hline$J_{m}$ & $\left(\mathrm{~kg} \cdot \mathrm{m}^{2}\right)$ & 0.02 \\
\hline$B_{m}$ & $(\mathrm{~N} \cdot \mathrm{m} \cdot \mathrm{s})$ & 0.015 \\
\hline$T_{n}$ & $(\mathrm{~N} \cdot \mathrm{m})$ & 26.50 \\
\hline$\Psi_{n}$ & $(\mathrm{~Wb})$ & 0.90 \\
\hline Power & $(\mathrm{kW})$ & 4.0 \\
\hline$I_{n}$ & $(\mathrm{~A})$ & 11.88 \\
\hline Rated speed, $\omega_{n}$ & $(\mathrm{rpm})$ & 1430 \\
\hline$\gamma$ & & 25.70 \\
\hline$V_{d c}$ & $(\mathrm{~V})$ & 600.0 \\
\hline$K_{p}$ & & 2.0 \\
\hline$K_{i}$ & & 20.0 \\
\hline$T_{s}$ & $(\mu \mathrm{s})$ & 100.0 \\
\hline Simulation step & $(\mu \mathrm{s})$ & 1.0 \\
\hline
\end{tabular}
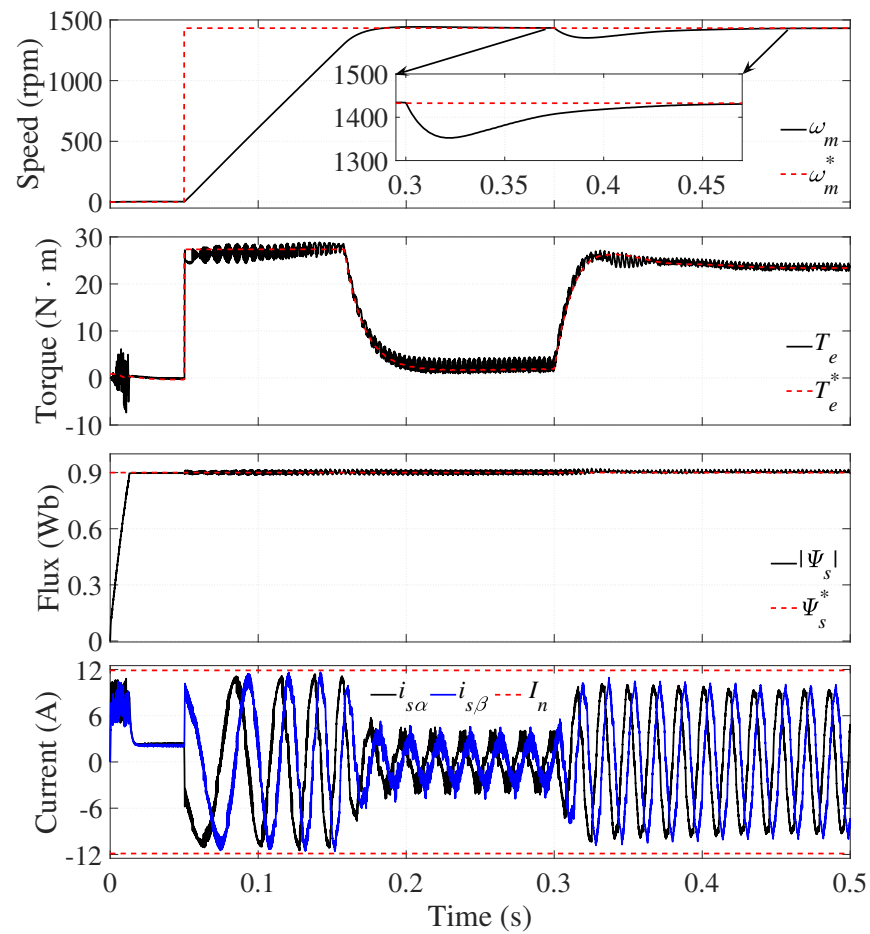

Fig. 4. Nominal speed step and load disturbance response of the developed PTC scheme. Speed, torque, stator flux and $\alpha-\beta$ stator current responses.

First, a step reference speed from static to the rated value is carried out. The speed, torque, flux and stator current are analysed as shown in Fig 4. The nominal air-gap flux is generated during the initial seconds with $\omega_{m}^{*}=0$. A fast flux response with good reference tracking, low ripple and $\alpha-\beta$ stator currents below $I_{n}$ are achieved during this interval. Then, $\omega_{m}^{*}$ is set to $\omega_{n}$ at $0.05(\mathrm{~s})$, while $T_{L}=0$. The nominal torque is produced quickly with a small ripple and without overcurrents. However, the stator flux ripple is slightly increased. The speed rise time (from 5\% to $95 \%$ of the target) is $0.108(\mathrm{~s})$. Once $\omega_{n}$ is reached, $T_{e}^{*}$ decreases up to the 
minimum required to maintain the speed under the friction effect. During the transient- and steady-state periods, the considered variables present adequate behaviour. A quick dynamic response can be perceived in this part of the evaluation.

A load torque of $75 \%$ of the rated magnitude is applied during the steady-state interval at 0.30 (s) to analyse the load disturbance effect. The speed zoom in the interval of 0.30 to 0.47 (s) shown in Fig. 4 depicts that $\omega_{m}$ only falls from $100 \%$ to $94.6 \%$ of $\omega_{n}$. The controller quickly generates a electromagnetic torque with a low ripple that accelerates the rotor of the machine and recovers the command speed in 0.15 (s). The stator flux is also regulated appropriately with a small ripple. Finally, the speed is again controlled to $\omega_{n}$ with a $T_{e}$ that maintains the equilibrium. The $\alpha-\beta$ stator current does not surpass $I_{n}$ during the described operation due to the penalisation implemented in (10).

The previous evaluation also demonstrates the proper operation of the overcurrent protection. An assessment of the $i_{s 1}$ phase current quality in the time and frequency domains is depicted in Fig. 5. A capture from the previous test of two cycles at steady-state shows the voltage waveform with the fixed switching frequency pattern generated by the controller. A quasi sinusoidal current response is achieved in the time domain. This result is confirmed in the frequency response. The current spectrum depicts the low energy retained by undesired harmonic components. Only small currents are perceived with the zoom in the low-frequency interval and the sampling frequency neighbourhood. The fundamental component is 9.21 (A), whereas total harmonic distortion $\mathrm{THD}_{i}$ is $4.34 \%$.

Figure 6 shows the results achieved with the fixed switching frequency PTC during the application of a no-load reversal speed test from $\omega_{n}$ to $-\omega_{n}$. The speed change is finished in 0.24 (s). Again, the scheme provides a fast torque/flux response with low ripple and current magnitudes inside of the nominal band. The operation demonstrates the appropriate behaviour of the regulated variables in the low-speed region and the zero speed crossing.

Finally, a performance comparison between the classic [8] and the proposed PTC strategies at the same average switching frequency is conducted. A torque load of $85 \%$ of the nominal valued is applied with the machine controlled at different speeds. The conventional scheme [8] is implemented with a sampling frequency $f_{s}$ of $20(\mathrm{kHz})$, and the average switching frequencies attained in the analysed operation points are measured. Then, the introduced PTC is configured to operate at the evaluated speeds, providing the same number of commutations than the traditional scheme. Figure 7 shows the torque and flux mean squared errors (top and middle, respectively) along with the $\mathrm{THD}_{i}$ (bottom) obtained with both techniques. The classic method produces practically constant mean squared errors and $\mathrm{THD}_{i}$ under the test conditions. However, the spectrum reports a small amount of energy distributed in a wide band of harmonic frequencies and average switching frequencies between $1 / 10$ and $1 / 5$ of $f_{s}$. On the other hand, the proposed PTC overcomes the last issue at the expense of slightly worse torque/flux tracking and current distortion.
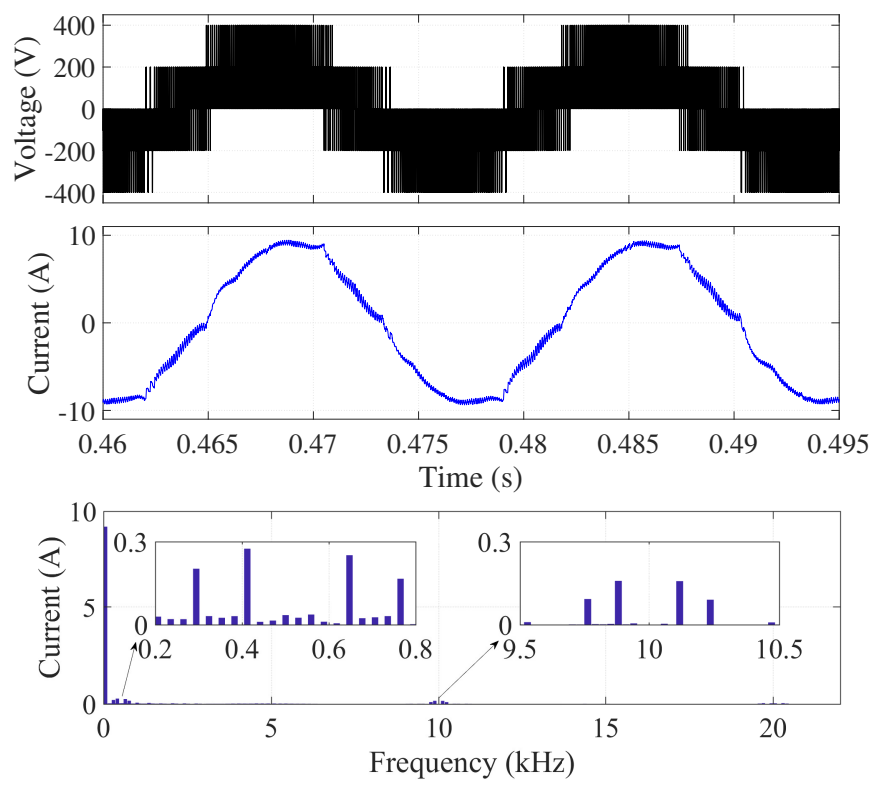

Fig. 5. Steady-state $v_{s 1}$ voltage waveform along with the response of $i_{s 1}$ phase current in the time and frequency domains.
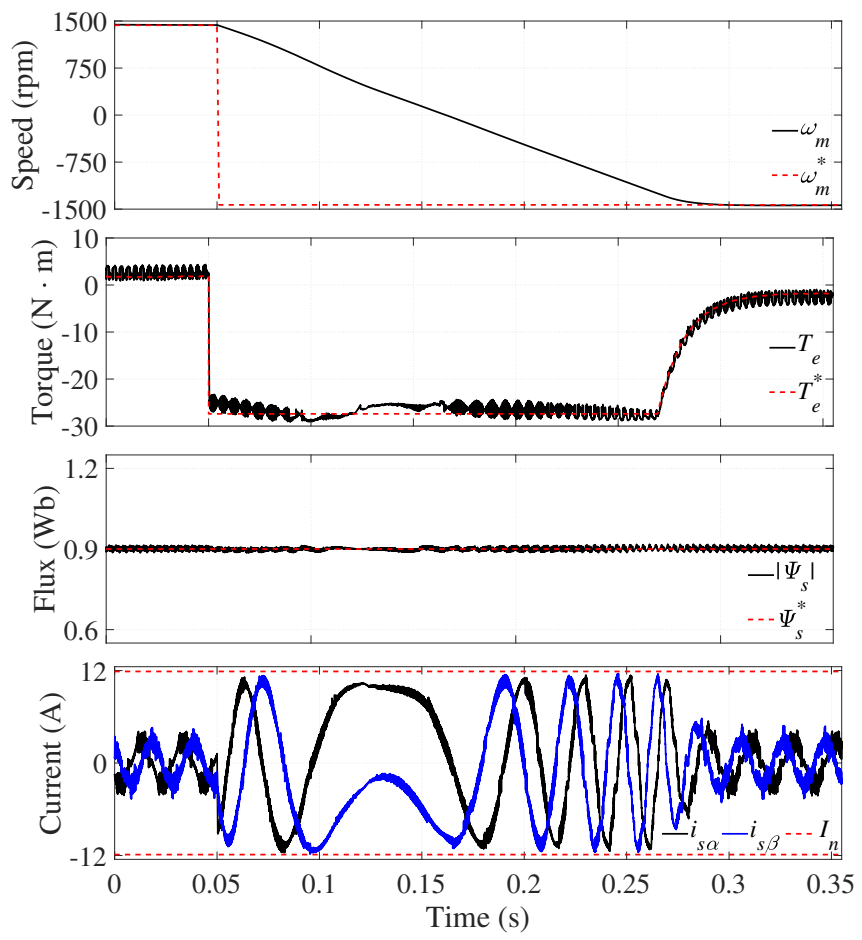

Fig. 6. Performance of the proposed PTC under a reversal speed test. Speed, torque, stator flux and $\alpha-\beta$ stator current responses.

Notice that the conditions of the latest tests were used only to carry out the comparison. The proposed PTC operated with a sampling frequency range between 2 to $3.9(\mathrm{kHz})$. This condition is unfavourable for predictive control schemes based on forward Euler approach. The method could be easily implemented with smaller $T_{s}$ periods in modern digital controllers to achieve better responses like the shown in Fig. 4. 


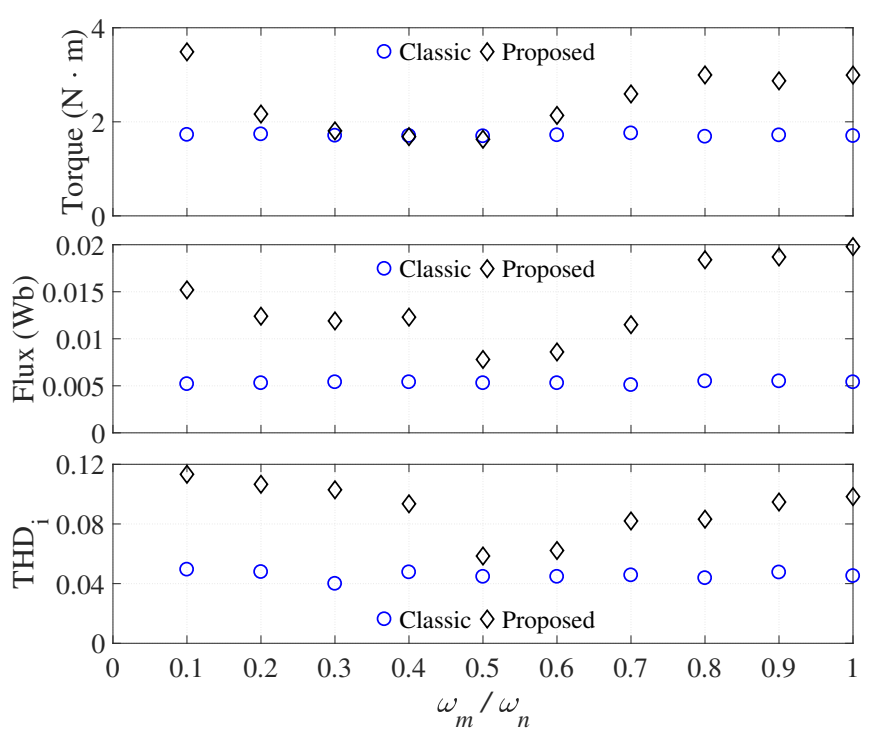

Fig. 7. Comparison between the classic and the developed PTC schemes at different speeds with the same average switching frequency. Torque and stator flux mean squared errors together with the $\mathrm{THD}_{i}$.

\section{CONCLUSiON}

A novel predictive torque control strategy for induction motor drives with fixed switching frequency has been introduced in this paper. The architecture of the classic controller has redesigned to select an optimum pair of active voltage vectors with their dwell times. The solution is applied with the well-known seven-segment switching pattern to achieve a constant number of commutations during a sampling period. The resulted scheme demonstrates a fast and good torque/flux tracking with a low stator current distortion and overcurrent protection. The power spectrum drawback of predictive control techniques is mitigated at the expense of a slightly higher torque/flux mean squared errors and current distortion compared to the classic implementation at the same average switching frequency.

\section{ACKNOWLEDGMENT}

The authors would like to thank the financial support provided by the FONDECYT Regular 1160690 and Postdoctoral 3170014 research projects for the development of this work.

\section{REFERENCES}

[1] X. Zhang and G. H. B. Foo, "Overmodulation of constant-switchingfrequency-based DTC for reluctance synchronous motors incorporating field-weakening operation," IEEE Trans. Ind. Electron., vol. 66, no. 1, pp. 37-47, Jan 2019.

[2] K. M. R. Eswar, K. V. P. Kumar, and T. V. Kumar, "A simplified predictive torque control scheme for open-end winding induction motor drive," IEEE J. of Emerg. Sel. Topics in Power Electron., vol. 7, no. 2, pp. 1162-1172, June 2019.

[3] P. Karamanakos and T. Geyer, "Model predictive torque and flux control minimizing current distortions," IEEE Trans. Power Electron., vol. 34, no. 3, pp. 2007-2012, March 2019.

[4] F. Wang, H. Xie, Q. Chen, S. A. Davari, J. Rodriguez, and R. Kennel, "Parallel predictive torque control for induction machines without weighting factors," IEEE Trans. Power Electron., 2019. DOI 10.1109/TPEL.2019.2922312.

[5] I. M. Alsofyani, N. R. N. Idris, and K. Lee, "Dynamic hysteresis torque band for improving the performance of lookup-table-based DTC of induction machines," IEEE Trans. Power Electron., vol. 33, no. 9, pp. 7959-7970, Sep. 2018

[6] F. Wang, Z. Zhang, S. A. Davari, R. Fotouhi, D. Arab Khaburi, J. Rodríguez, and R. Kennel, "An encoderless predictive torque control for an induction machine with a revised prediction model and EFOSMO,” IEEE Trans. Ind. Electron., vol. 61, no. 12, pp. 6635-6644, Dec 2014

[7] A. Salem, M. Mamdouh, and M. Abido, "Predictive torque control and capacitor balancing of a SiC-based dual T-type drive system," IEEE Trans. Power Electron., 2019. DOI 10.1109/TPEL.2019.2930883.

[8] H. Miranda, P. Cortes, J. I. Yuz, and J. Rodriguez, "Predictive torque control of induction machines based on state-space models," IEEE Trans. Ind. Electron., vol. 56, no. 6, pp. 1916-1924, June 2009.

[9] P. Guazzelli, W. de Andrade Pereira, C. de Oliveira, A. de Castro, and M. de Aguiar, "Weighting factors optimization of predictive torque control of induction motor by multiobjective genetic algorithm," IEEE Trans. Power Electron., vol. 34, no. 7, pp. 6628-6638, July 2019.

[10] M. Rivera, D. Faundez, J. Kolar, P. Wheeler, J. A. Riveros, and S. Toledo, "Three-phase rectifiers with current compensation schemes - part i: Passive circuits," in 2018 IEEE International Conference on Electrical Systems for Aircraft, Railway, Ship Propulsion and Road Vehicles International Transportation Electrification Conference (ESARS-ITEC), Nov 2018, pp. 1-6.

[11] S. Chowdhury, P. Wheeler, Z. Huang, M. Rivera, and C. Gerada, "Fixed switching frequency predictive control of an asymmetric source dual inverter system with a floating bridge for multilevel operation," IET Power Electronics, vol. 12, no. 3, pp. 450-457, 2019.

[12] D. G. Holmes and T. A. Lipo, Pulse Width Modulation for Power Converters: Principles and Practice, 1st ed. Wiley-IEEE Press, Oct 2003, ch. 6, pp. 259-336.

[13] E. Fuentes, C. A. Silva, and R. M. Kennel, "MPC implementation of a quasi-time-optimal speed control for a PMSM drive, with inner modulated-FS-MPC torque control," IEEE Trans. Ind. Electron., vol. 63, no. 6, pp. 3897-3905, June 2016.

[14] F. Donoso, A. Mora, R. Cárdenas, A. Angulo, D. Sáez, and M. Rivera, "Finite-set model-predictive control strategies for a 3L-NPC inverter operating with fixed switching frequency," IEEE Trans. Ind. Electron., vol. 65 , no. 5, pp. 3954-3965, May 2018

[15] M. Mamdouh and M. A. Abido, "Efficient predictive torque control for induction motor drive," IEEE Trans. Ind. Electron., vol. 66, no. 9, pp. 6757-6767, Sep. 2019. 